\title{
Clinical Utility of CAI25 Levels in Predicting Laparoscopically Confirmed Salpingitis in Patients With Clinically Diagnosed Pelvic Inflammatory Disease
}

\author{
E. Moore and D.E. Soper* \\ Department of Obstetrics and Gynecology, Medical College of Virginia, Virginia Commonwealth \\ University, Richmond, VA
}

\begin{abstract}
Objective: The purpose of this study was to determine the utility of serum CA125 determinations in diagnosing acute salpingitis.

Methods: CA125 levels were determined for 34 women with the clinical diagnosis of pelvic inflammatory disease (PID). Acute salpingitis was confirmed laparoscopically in 28 women (82.3\%).

Results: Twenty patients (71.4\%) with laparoscopically confirmed acute salpingitis had CA125 levels greater than 7.5 units, compared with no patients $(0 / 6)$ with laparoscopically normal tubes $(P$ $=0.002$ ). The degree of elevation of CA125 levels correlated with the severity of tubal inflammation noted at laparoscopy. All patients with levels above 16 units had laparoscopically severe salpingitis.

Conclusions: We conclude that while CA125 levels above 7.5 units may modestly improve the ability of the clinical diagnosis of PID to accurately reflect visually confirmed acute salpingitis, limitations of the test make its clinical utility questionable. Infect. Dis. Obstet. Gynecol. 6:182-185, 1998. ๑ 1998 Wiley-Liss, Inc.
\end{abstract}

$\mathrm{T}$ he glycoprotein CA125, a cell surface antigen recognized by the OC-125 murine monoclonal antibody, has become widely used clinically in gynecologic oncology. Bast first introduced its use as a marker for ovarian cancer in $1983 .{ }^{1}$ Since that time, it has become apparent that CA125 is not $100 \%$ specific for epithelial ovarian carcinoma. Other gynecologic and nongynecologic cancers, endometriosis, pregnancy, uterine myomas, and pelvic inflammatory disease (PID) have all been found to be associated with an elevated level of CA125. ${ }^{2}$

PID continues to pose a diagnostic dilemma for gynecologists. Clinical criteria alone do not bear a relationship to the severity of salpingitis as graded laparoscopically. ${ }^{3,4}$ In addition, it is now recognized that the clinical symptoms and signs of PID are varied, and some women may actually have "silent salpingitis." Because of the long-term sequelae of this disease (infertility, ectopic pregnancy, chronic pain, pelvic abscesses, and sexual dysfunction), prompt diagnosis and appropriate treatment are imperative. A laboratory marker for tubal inflammation would be of great benefit clinically in helping to diagnose and therefore prompt treatment of this disease.

The CA125 antigen is found in the normal adult fallopian tube epithelium, raising the possibility that it may be a useful marker for the clinical di-

*Correspondence to: Dr. David E. Soper, Department of Ob/Gyn, Medical University of South Carolina, 171 Ashley Avenue, Charleston, SC 29425. 
agnosis of salpingitis. Previous investigations looking at the usefulness of CA125 determinations as a screening test for ovarian cancer have noted that CA125 levels may be elevated in women with PID. CA125 levels have been studied specifically as a tool for their predictive value in diagnosing PID and detecting the degree of inflammation when PID is suspected. ${ }^{5-7}$ None of these studies have been performed on a U.S. population of patients.

The purpose of this study was to evaluate the ability of a serum CA125 level to confirm the presence of laparoscopically visualized salpingitis and to correlate CA125 levels with the severity of laparoscopic findings in patients from an inner city emergency room population with the clinical diagnosis of PID.

\section{MATERIALS AND METHODS}

The study population consisted of 34 patients with clinically suspected PID who were admitted to the gynecology service through the emergency room at the Medical College of Virginia. Each patient's age, race, gravidity and parity, and history of sexually transmitted diseases were recorded at the time of admission. All patients had CA125 levels drawn and underwent laparoscopy at the time of admission.

Criteria for the clinical diagnosis of PID were pelvic pain, the presence of bilateral adnexal tenderness during bimanual pelvic examination, and the presence of leukorrhea by microscopy of the vaginal secretions. Patients who were entered into the study were clinically graded as to severity of illness at the time of admission. Patients were considered to have clinically severe PID if they presented with a temperature $>101^{\circ} \mathrm{F}$ and/or a white blood cell count $>15,000 / \mathrm{mL}$; otherwise, they were clinically graded as mild.

Levels of CA125 antigen were determined with an assay kit supplied by Centocor according to the manufacturer's instructions. Total assay time is approximately 26 hours.

Each patient admitted to the study underwent laparoscopic diagnosis and, if found, grading of salpingitis. Minimal criteria for the laparoscopic confirmation of acute salpingitis included tubal erythema, tubal edema, and purulent exudate either on the tube, from the ostia, or in the cul de sac. Severity of disease was graded according to the crite-
TABLE I. Demographic variables

\begin{tabular}{|c|c|c|c|}
\hline Variable & $\begin{array}{c}\text { Acute } \\
\text { salpingitis } \\
(\mathrm{N}=28)\end{array}$ & $\begin{array}{c}\text { Control } \\
\text { (normal tubes) } \\
(\mathrm{N}=6)\end{array}$ & $P$ value \\
\hline Age (years) & $24.2 \pm 6.9$ & $24.2 \pm 3.3$ & $N S^{a}$ \\
\hline Race & & & 0.009 \\
\hline Black & 24 & 2 & \\
\hline White & 4 & 3 & \\
\hline Other & 1 & 0 & \\
\hline Gravidity & $2.1 \pm 1.7$ & $1.2 \pm 1.6$ & NS \\
\hline Parity & $1.0 \pm 1.1$ & $0.5 \pm 0.8$ & NS \\
\hline History of $\mathrm{GC}^{\mathrm{b}}$ & 18 & 1 & 0.07 \\
\hline History of PIDC & 6 & 2 & NS \\
\hline
\end{tabular}

${ }^{a} \mathrm{NS}$, not significant.

${ }^{b} \mathrm{GC}$, gonorrhea.

CPID, pelvic inflammatory disease.

ria of Westrom and Mardh. ${ }^{8}$ Mild salpingitis was therefore defined as meeting the above minimum criteria with freely mobile tubes and patent ostia (grade 1). Moderate salpingitis was defined as tubes with more pronounced inflammation, fibrinous exudates on the serosal surfaces, and tubes which are not freely mobile (grade 2). Severe salpingitis was defined by the presence of tuboovarian abscess or adherence of pelvic organs to one another (grade 3 ).

\section{STATISTICAL ANALYSIS}

Categorical variables were analyzed using the chisquare or Fisher exact test as appropriate. Continuous variables were analyzed using the paired $t$-test. All $P$ values were calculated based on two-tailed tests with $P<0.05$ considered significant.

\section{RESULTS}

The demographics of the patient population studied are shown in Table 1 . The mean age of the study population was 24.2 years. Most patients entered into the study were African American (26/34). There was no statistically significant difference between the group of patients found to have normal tubes and those with salpingitis with respect to age, gravidity, parity, history of gonorrhea, or history of PID.

Parameters significantly different between the salpingitis and no salpingitis (control) group included admission temperature, admission status, and C-reactive protein levels. Mean white blood cell count and mean CA125 levels also varied between the two groups, but these values failed to 
TABLE 2. Clinical variables

\begin{tabular}{lccc}
\hline & $\begin{array}{c}\text { Acute } \\
\text { Salpingitis }\end{array}$ & $\begin{array}{c}\text { Control } \\
\text { (normal } \\
\text { tubes) }\end{array}$ & $P$ value \\
\hline Temperature $\left({ }^{\circ} \mathrm{F}\right)$ & $100.9 \pm 1.8$ & $99.2 \pm 0.6$ & 0.0009 \\
White blood cell count & & & \\
$\quad\left(1000 / \mathrm{m}^{3}\right)$ & $13.7 \pm 6.1$ & $8.2 \pm 2.8$ & 0.06 \\
C-reactive protein $(\mathrm{mg} / \mathrm{dL})$ & $8.5 \pm 7.7$ & $1.0 \pm 0.0$ & 0.008 \\
CAI25 (units) & $28.9 \pm 60.6$ & $7.5 \pm 0.0$ & 0.07 \\
\hline
\end{tabular}

reach statistical significance when using the Student $t$-test for continuous variables. (Table 2).

When using a chi-square test comparing the group with CA125 levels $>7.5$ units and the group with levels $\leq 7.5$ units, there was statistical significance between the two groups $(P=0.002)$ (Table $3)$. Of the 34 patients recruited into the study, 28 $(82.3 \%)$ were found to have salpingitis at laparoscopy. Of the 28 patients with salpingitis, 20 had CA125 levels above 7.5 units, seven had levels above 16 units, and four had levels above 35 units. Of the six patients with no salpingitis at laparoscopy, none had CA125 levels above 7.5 units. All women with CA125 levels above 16 units had visually graded severe (grade 3 ) salpingitis by laparoscopy. CA125 levels increased with an increasingly severe grade of salpingitis (Table 4).

Using 7.5 units as the cutoff for a normal CA125 level, the sensitivity of an abnormal level predicting acute salpingitis in this population was $71 \%$. The specificity of a CA125 level >7.5 units was $100 \%$. CA125 was not elevated in eight patients with laparoscopic salpingitis. Predictive negative value was therefore only $43 \%$.

The pretest probability of a patient in this select population having salpingitis was 0.82 . The likelihood ratio of patients with CA125 levels $>7.5$ units having salpingitis compared with no salpingitis is 20/28:0/28. ${ }^{9}$ This cannot be calculated since the denominator becomes zero. However, if we consider a single patient with CA125 >7.5 units without PID, as may occur with a larger study population, the likelihood ratio becomes $20 / 28: 1 / 28$ or 20 . Using Fagan's nomogram, the posttest probability of PID is $>95 \%$ when a patient meeting the clinical criteria for PID also has a CA125 level of >7.5. In other words, the predictive value of the clinical diagnosis alone versus the clinical diagnosis plus a CA125 > 7.5 units improved from $82 \%$ to more than $95 \%$ in diagnosing acute salpingitis.
TABLE 3. CAI 25 as a predictor of salpingitis at laparoscopy

\begin{tabular}{lcc}
\hline & $\begin{array}{c}\text { Patients with } \\
\text { salpingitis }\end{array}$ & $\begin{array}{c}\text { Patients without } \\
\text { salpingitis }\end{array}$ \\
\hline CAI25 $\leq 7.5$ units & 8 & 6 \\
CAI $25>7.5$ units & 20 & 0 \\
\hline
\end{tabular}

TABLE 4. Correlation of grade of salpingitis with CAI 25 level

\begin{tabular}{lc}
\hline Grade $^{\mathrm{a}}$ & CAI25 level (units) \\
\hline 0 & $7.5 \pm 0.0$ \\
1 & $8.6 \pm 3.1$ \\
2 & $11.0 \pm 4.2$ \\
3 & $65.1 \pm 93.4$ \\
\hline
\end{tabular}

${ }^{a} G$ rade I vs. $3, P=0.09$; grade 0 vs. $3, P=0.08$; grade 0 vs. $2, P=$ not significant.

\section{DISCUSSION}

Pelvic inflammatory disease is estimated to affect approximately 1 million women a year for a total annual cost of $\$ 3.5$ billion. It is a cause of significant morbidity in the female population. Salpingitis increases the risk of ectopic pregnancy by six- to tenfold, increases the risk for chronic pelvic pain, causes infertility in $11 \%$ of women with their first infection, and increases the risk of future PID, leading to even higher levels of infertility with each subsequent infection. PID tends to be readily treatable with antibiotics when properly diagnosed; therefore, the key to decreasing the morbidity from this disease is in the diagnosis.

Unfortunately, salpingitis is not easily diagnosed by clinical exam, routine laboratory tests, or noninvasive imaging studies. The gold standard for the diagnosis of salpingitis is laparoscopy, but it is not practical to operate on every patient for whom the diagnosis of PID is considered. A test that would increase the likelihood of a particular subset of patients as having PID, and that would be able to exclude those unlikely to have an upper genital tract infection, would be extremely valuable for the management of this illness. CA125 levels have been proposed for use as a laboratory marker for salpingitis.

In the group of patients studied, 7.5 units seems to be the most logical CA125 level to use as a cutoff for a normal CA125 level. Bast et al. noted in their initial article that "the choice of limits for normal 
values is arbitrary." 1 Thirty-five units and 65 units were chosen as potential cutoff values for normal when looking for ovarian cancer because only $1 \%$ and $0.2 \%$, respectively, of their normal population had levels above these values. Since that test was being evaluated for its utility in screening for ovarian cancer in the population as a whole, a high cutoff value for normal was chosen to increase the specificity of the test. The investigators needed high specificity because of the low incidence of the targeted disease (ovarian cancer) within the general population. Other authors have chosen 16 units or 30 units as their cutoff level when looking at CA125 and salpingitis in select groups of patients. $^{5,6}$

Only 3 patients $(10.7 \%)$ in this study with acute salpingitis by laparoscopy had a level of CA125 greater than 35 units. No patients with a CA125 level over 7.5 units had normal tubes at laparoscopy. A higher cutoff level, at least in this particular population, lowers the sensitivity of the test without improving specificity, since one does not weed out any false positives and only adds false negatives. In this population, 7.5 units makes the most sense as a cutoff value to optimize the utility of the test.

The main limitation of this study is that the population tested is not the actual population one would like to have screened. In an emergency room or office situation, the population in which a diagnostic test would be helpful is that population presenting with more atypical signs of PID. $\mathrm{Pa}-$ tients with abdominal pain but no mucopus, mucopus but no cervical motion tenderness, or irregular bleeding of unclear etiology would all be populations for whom a diagnostic test to detect the presence of salpingitis would be of value. Such a patient population would clearly have a lower incidence of PID and therefore challenge further the ability of CA125 to diagnose patients with true salpingitis. In this study's high-incidence population, CA125 appears to be correlated with the presence of salpingitis, but these are women for whom the clinical decision to admit and treat has already been made. One might expect CA125 to perform less well in a population with a lower incidence of disease.

As well as appearing only modestly helpful in improving the clinical diagnosis of PID in predict- ing salpingitis, the test for CA125 also poses a practical problem that would need to be addressed before it could be used clinically. Currently, the laboratory technique requires a 24-hour incubation period, precluding the rapid availability of results needed in an office or emergency room setting.

This study suggests that serum CA125 determinations modestly improve the predictive value of the clinical diagnosis of PID to accurately reflect the presence of acute salpingitis. Because the predictive negative value of the test appears to be low, however, it does not seem that CA125 levels could be used to rule out salpingitis. CA125 might be useful to investigators looking for a way to rule in salpingitis without laparoscopy in a study population.

\section{REFERENCES}

1. Bast RC Jr, Klug TL, St John E, Jenison E, et al.: A radioimmunoassay using a monoclonal antibody to monitor the course of epithelial ovarian cancer. $\mathrm{N} \mathrm{Engl}$ J Med 309:883-887, 1983.

2. Jacobs I, Bast RC Jr: The CA125 tumor-associated antigen: A review of the literature. Hum Reprod 4:1-12, 1989.

3. Soper DE, Brockwell NJ, Dalton HP: Microbial etiology of urban emergency department acute salpingitis: Treatment with ofloxacin. Am J Obstet Gynecol 167: 653-660, 1992.

4. Svensson L, Westrom L, Ripa KT, Mardh P-A: Differences in some clinical and laboratory parameters in acute salpingitis related to culture and serologic findings. Am J Obstet Gynecol 138:1017-1021, 1980.

5. Duk JM, Kauer FM, Fleuren GJ, de Bruijn HW: Serum CA125 levels in patients with a provisional diagnosis of pelvic inflammatory disease. Clinical and theoretical implications. Acta Obstet Gynecol Scand 68:637-641, 1989.

6. Paavonen J, Miettinen A, Heinonen PK, Aaran RK, et al.: Serum CA125 in acute pelvic inflammatory disease. Br J Obstet Gynaecol 96:574-579, 1989.

7. Brumstead JR, Mead PB, Gibson M: Serum concentration of protein CA125 in women with pelvic inflammatory disease. Sex Transm Dis 15:144-147, 1988.

8. Westrom L., Mardh P-A: Salpingitis. In Holmes KK, Mardh P-A, Sparling PF, et al. (eds.): Sexually Transmitted Diseases. 1st ed. New York: McGraw-Hill, pp 615-632, 1984.

9. Jaeschke R, Guyatt GH, Sackett DL: Users' guide to the medical literature. III. How to use an article about a diagnostic test. B. What are the results and will they help me in caring for my patients? JAMA 271:703-707, 1994. 


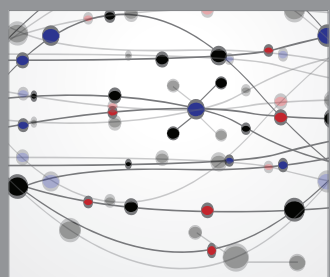

The Scientific World Journal
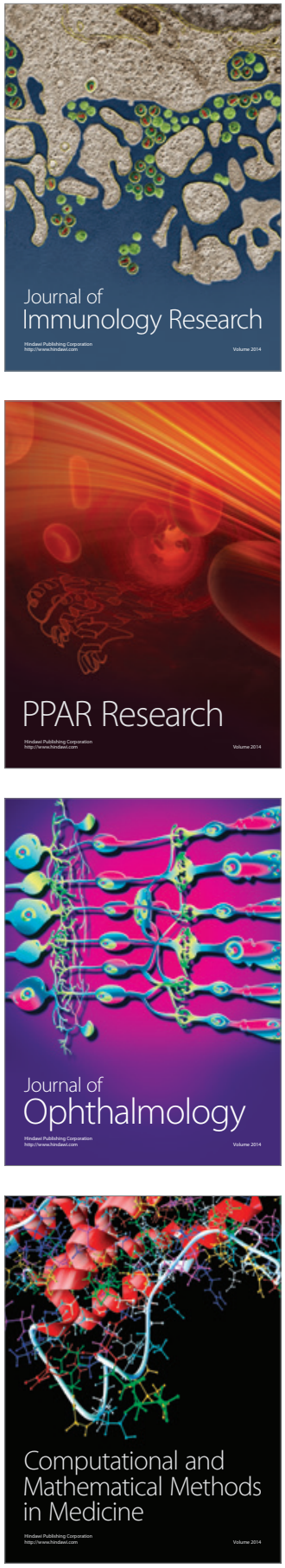

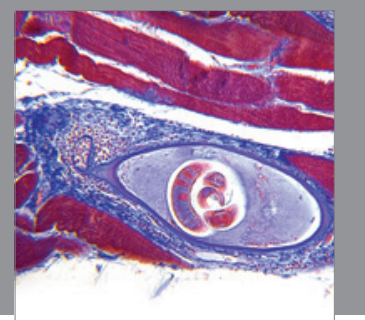

Gastroenterology

Research and Practice
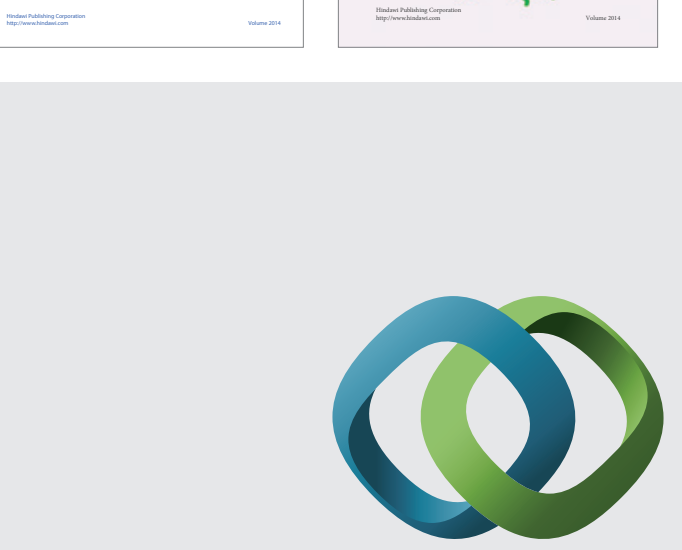

\section{Hindawi}

Submit your manuscripts at

http://www.hindawi.com
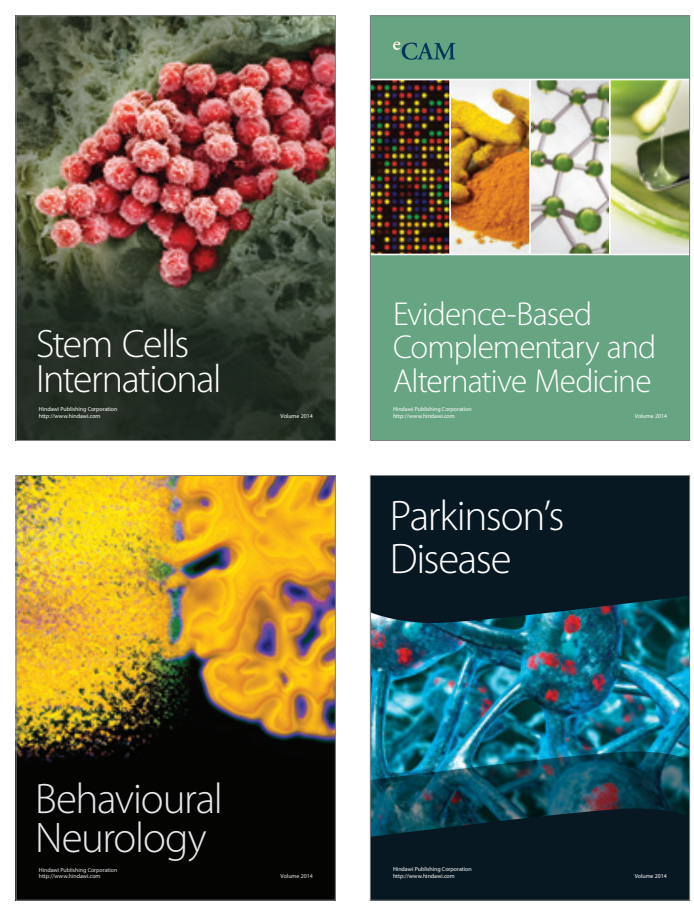

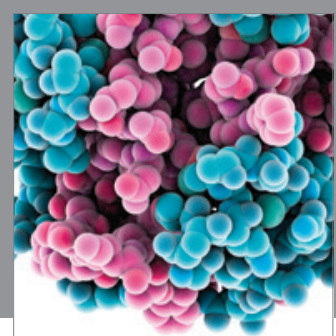

Journal of
Diabetes Research

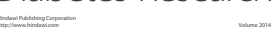

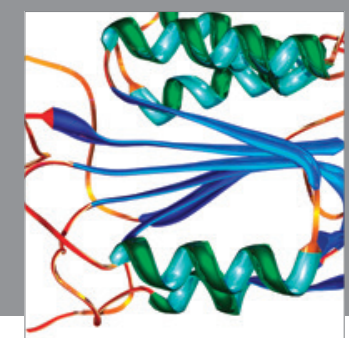

Disease Markers
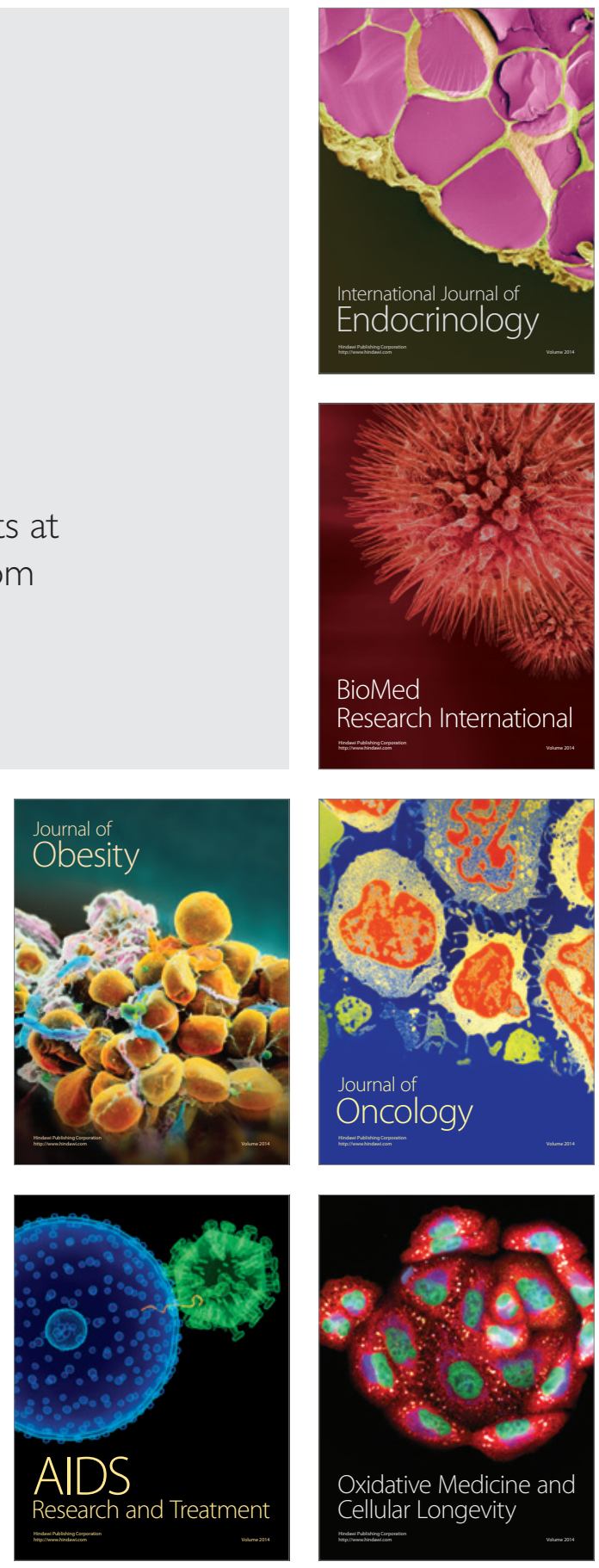